\section{OPEN ACCESS}

Edited by:

Valentina Tomassini,

University of Studies G. d'Annunzio

Chieti and Pescara, Italy

Reviewed by:

Shannon Kolind,

University of British Columbia, Canada

Antonio Giorgio,

University of Siena, Italy

*Correspondence:

Zsigmond Tamás Kincses

kincses.zsigmond.tamas@ med.u-szeged.hu

Specialty section:

This article was submitted to

Multiple Sclerosis and

Neuroimmunology,

a section of the journal

Frontiers in Neurology

Received: 19 October 2020

Accepted: 14 January 2021

Published: 03 March 2021

Citation:

Kocsis K, Szabó N, Tóth E, Király A,

Faragó $P$, Kincses $B$, Veréb $D$,

Bozsik B, Boross K, Katona M,

Bodnár P, László NG, Vécsei L,

Klivényi P, Bencsik K and Kincses ZT

(2021) Two Classes of T1 Hypointense

Lesions in Multiple Sclerosis With

Different Clinical Relevance.

Front. Neurol. 12:619135.

doi: 10.3389/fneur.2021.619135

\title{
Two Classes of T1 Hypointense Lesions in Multiple Sclerosis With Different Clinical Relevance
}

\begin{abstract}
Krisztián Kocsis ${ }^{1}$, Nikoletta Szabó ${ }^{1}$, Eszter Tóth ${ }^{1}$, András Király ${ }^{1}$, Péter Faragó ${ }^{1}$, Bálint Kincses ${ }^{1}$, Dániel Veréb ${ }^{2}$, Bence Bozsik ${ }^{1}$, Katalin Boross ${ }^{1}$, Melinda Katona ${ }^{3}$, Péter Bodnár ${ }^{3}$, Nyúl Gábor László ${ }^{3}$, László Vécsei ${ }^{1,4}$, Péter Klivényi ${ }^{1}$, Krisztina Bencsik ${ }^{1}$ and Zsigmond Tamás Kincses ${ }^{1,2 *}$
\end{abstract}

\begin{abstract}
${ }^{1}$ Department of Neurology, Albert Szent-Györgyi Clinical Center, University of Szeged, Szeged, Hungary, ${ }^{2}$ Department of Radiology, Albert Szent-Györgyi Clinical Center, University of Szeged, Szeged, Hungary, ${ }^{3}$ Department of Image Processing and Computer Graphics, University of Szeged, Szeged, Hungary, ${ }^{4}$ Magyar Tudományos Akadémia-Szegedi Tudományegyetem (MTA-SZTE) Neuroscience Research Group, Szeged, Hungary
\end{abstract}

Background: Hypointense lesions on T1-weighted images have important clinical relevance in multiple sclerosis patients. Traditionally, spin-echo (SE) sequences are used to assess these lesions (termed black holes), but Fast Spoiled Gradient-Echo (FSPGR) sequences provide an excellent alternative.

Objective: To determine whether the contrast difference between T1 hypointense lesions and the surrounding normal white matter is similar on the two sequences, whether different lesion types could be identified, and whether the clinical relevance of these lesions types are different.

Methods: Seventy-nine multiple sclerosis patients' lesions were manually segmented, then registered to T1 sequences. Median intensity values of lesions were identified on all sequences, then K-means clustering was applied to assess whether distinct clusters of lesions can be defined based on intensity values on SE, FSPGR, and FLAIR sequences. The standardized intensity of the lesions in each cluster was compared to the intensity of the normal appearing white matter in order to see if lesions stand out from the white matter on a given sequence.

Results: $100 \%$ of lesions on FSPGR images and 69\% on SE sequence in cluster \#1 exceeded a standardized lesion distance of $Z=2.3$ ( $p<0.05)$. In cluster \#2, 78.7\% of lesions on FSPGR and only $17.7 \%$ of lesions on SE sequence were above this cutoff value, meaning that these lesions were not easily seen on SE images. Lesion count in the second cluster (lesions less identifiable on SE) significantly correlated with the Expanded Disability Status Scale (EDSS) $(R: 0.30, p \leq 0.006)$ and with disease duration $(R: 0.33, p$ $\leq$ 0.002).

Conclusion: We showed that black holes can be separated into two distinct clusters based on their intensity values on various sequences, only one of which is related to clinical parameters. This emphasizes the joint role of FSPGR and SE sequences in the monitoring of MS patients and provides insight into the role of black holes in MS.

\footnotetext{
Keywords: multiple sclerosis, T1 hypointense lesions, black holes, MRI protocol, clustering, clinical state
} 


\section{INTRODUCTION}

Multiple sclerosis (MS) is a chronic, progressive disease affecting the central nervous system in young adults leading to demyelination and axonal loss (1). Conventional T1 (T1w)and $\mathrm{T} 2$-weighted (T2w) magnetic resonance (MR) imaging has a key role in the diagnosis and in the follow-up of MS, but the relationship between clinical symptoms and white matter lesions is not straightforward (2). White matter lesions on T2w images are hyperintense and correspond to several histopathological changes such as edema or demyelination (3).

In contrast, hypointense white matter lesion on T1w images, the so-called black holes (BHs) correspond to axonal loss (4). The first description of the association between BHs and the clinical state of MS patients was published by Truyen et al. Using spin-echo (SE) MRI sequences in their 40-month followup study they showed that patients' baseline disabilities correlated significantly with $\mathrm{BH}$ lesion load, and in the case of secondary progressive $\mathrm{MS}$, the accumulation of $\mathrm{BHs}$ contributed to the progression rate as well. They suggested that the presence of $\mathrm{BHs}$ led to a failed remission (5). BHs could be divided into two major types, namely, acute and persisting $\mathrm{BHs}$. Acute $\mathrm{BH}$ are contrast-enhancing lesions, and during the course of the disease, $20-40 \%$ of the acute BHs turns into persisting BHs $(4,6)$. $\mathrm{BHs}$ have high water content and correspond to irreversible MS pathology (7-10). In addition, the pattern of spatial distribution of BHs has preferred loci in the brain; for example, BHs are rarely present around the lateral ventricles but appear more often in the supratentorial region, with an effect on supratentorial brain volume and consequently cerebral atrophy (11). It was also reported that $\mathrm{BHs}$ may play a role in the conversion of clinically isolated syndrome to MS (12).

Many studies investigated the possible contribution of $\mathrm{BHs}$ to the clinical and cognitive state or their worsening in patients with MS. Based on these studies, BHs may present a possible resolution to the clinico-radiological paradox. Cognitive dysfunctions, reported in $43-70 \%$ of MS patients, mainly affect the information processing speed, visuospatial memory, and executive functions $(13,14)$. Parietal and frontal BHs were shown as a significant predictor of attention and non-verbal intelligence as well as phonemic verbal fluency deficit (7). Giorgio et al. demonstrated in a follow-up study that $\mathrm{BH}$ lesion load and lesion count were associated not just with the baseline EDSS scores (which quantifies and monitors the disability of MS patients), but also with its worsening over a 10-year period (8).

After it was established that BHs contribute to the course and progression of the disease and have clinical relevance in MS, the main focus of researches was directed toward whether any variation in $\mathrm{BH}$ appearance could have clinical relevance. There are only a few studies conducted in this regard. Adusumilli et al. showed that $\mathrm{BH}$ and gray holes (e.g., less hypointense lesions), presumably measured on SE images can be differentiated with high reliability and correlated well with different clinical and cognitive measures (15). Tam in another study proved that $\mathrm{BHs}$ with lower intensity compared to other $\mathrm{BH}$ s have higher clinical relevance (16).
Importantly, the correlation between $\mathrm{T} 1$ hypointense lesions to pathological features and clinical symptoms was mainly investigated on SE T1 weighted images. The downside of this acquisition technique is the relatively longer acquisition time. Fast spoiled gradient-echo (FSPGR) sequences are suitable alternatives providing shorter acquisition times and/or improved resolution. Hypointense lesions are also detectable on FSPGR sequences, but the clinical relevance and the possible differences in comparison to SE sequences have not been thoroughly studied.

In this study, we aimed (i) to determine whether the contrast difference between white matter hypointensities and the surrounding normal white matter is similar on SE and FSPGR images, (ii) if two different lesion types could be identified based on the intensities on the two sequences, and (iii) if the clinical relevance of the two lesion types are different.

\section{METHODS}

\section{Patients}

In this study, 79 patients with relapsing-remitting MS (RRMS) diagnosis were enrolled. Patients were recruited from the Multiple Sclerosis Outpatient Clinic at the Department of Neurology, University of Szeged. Clinical and demographic parameters of the patient population are depicted in Table $\mathbf{1 .}$ All patients were in stable clinical state, no relapses occurred 6 months preceding and following the study. This study was approved by the Ethical Committee of the University of Szeged (Ref.No.: 000002/2016/OTIG). All study participants gave their written informed contribution in accordance with the Declaration of Helsinki.

\section{MR Imaging Acquisition}

MR imaging was performed on a 3 T GE Discovery 750w MR Scanner (GE Healthcare, Chalfont St. Giles, UK). In total, MR images from 79 relapsing-remitting MS patients were acquired as part of the routine clinical follow-up based on our recent recommendation (17). In the analysis, the following sequences were used: high-resolution $\mathrm{T} 1$ weighted anatomical images [3D spoiled gradient-echo images with inversion recovery $3 \mathrm{D}$ FSPGR IR: echo time (TE): $5.4 \mathrm{~ms}$; repetition time (TR): $2 \mathrm{~ms}$; inversion time: $450 \mathrm{~ms}$; matrix: $256^{*} 256$; field of view (FOV): $25.6 \times 25.6 \mathrm{~cm}$; flip angle:12 degree; slice thickness: $1 \mathrm{~mm}$; PURE intensity correction, NEX:1], CUBE T2 FLAIR for lesion detection (TE: $135 \mathrm{~ms}$; TR: 6,700 ms; TI: 1,827 ms; matrix: $256 \times$ 224; FOV: $25 \times 22.5 \mathrm{~cm}$; slice thickness: $1.4 \mathrm{~mm}$; post-processing: ZIP512, ZIP2, NEX:1), SE T1 weighted images (TE: min fullminimum TE without fractional TE; TR: $500 \mathrm{~ms}$; flip angle: 73 degree; matrix: $256 \times 224$; FOV: $24 \times 19.2 \mathrm{~cm}$; slice thickness: $3 \mathrm{~mm}$; NEX:2).

\section{Image Analysis}

All the image analyses were carried out using the tools of the FSL software package (https://fsl.fmrib.ox.ac.uk/fsl/fslwiki/ FSL). All image (SE, FSPGR, and FLAIR) sequences were intensity normalized prior to the following steps (18). After removing the non-brain parts using BET (19), manual lesionoutlining on FLAIR images for all patients was performed by 
TABLE 1 | Demographic and clinical data of the patients.

\begin{tabular}{|c|c|c|c|c|c|c|c|}
\hline $\begin{array}{l}\text { Number of } \\
\text { patients }\end{array}$ & Females & $\begin{array}{c}\text { Age (years) } \\
\text { (mean } \pm \text { std) }\end{array}$ & $\begin{array}{c}\text { EDSS } \\
\text { (median } \pm \text { range) }\end{array}$ & $\begin{array}{l}\text { Disease duration } \\
\text { (years) (mean } \pm \text { std) }\end{array}$ & $\begin{array}{l}\text { Number of relapses since } \\
\text { treatment onset; median } \\
\text { (range) }\end{array}$ & Treatment regimen & $\begin{array}{c}\text { Lesion load }\left(\mathrm{mm}^{3}\right) \\
(\text { mean } \pm \text { std })\end{array}$ \\
\hline \multirow[t]{7}{*}{79} & 55 & 42.27 & 2 & 12.34 & $0(0-10)$ & DF-5.5\% & 7.09 \\
\hline & & $( \pm 9.89)$ & $(0-6)$ & $( \pm 7.44)$ & & Te-26.5\% & $( \pm 8.738)$ \\
\hline & & & & & & IFNb-12.5\% & \\
\hline & & & & & & GA-25\% & \\
\hline & & & & & & $\mathrm{F}-11.5 \%$ & \\
\hline & & & & & & A-12.5\% & \\
\hline & & & & & & N-6.5\% & \\
\hline
\end{tabular}

DF, dimethyl fumarate; Te, teriflunomide; IFNb, interferon beta 1a; GA, glatiramer acetate; F, fingolimod; A, alemtuzumab; N, natalizumab.

KK and supervised by KZT. In order to analyze only those lesions that are detectable on the $3 \mathrm{~mm}$ slice thickness images with certainty, lesions larger than 100 voxels were used in the analyses. SE and FLAIR images were registered to $3 \mathrm{D}$ FSPGR IR images with 6 DOF using FLIRT (20). The manually outlined and lesion masks were brought into FSPGR space using the previous registration matrices and trilinear interpolation. Masks were thresholded at 0.5 and binarized to avoid the enlargement due to the interpolation. Intensity values under the individual lesion masks on both FSPGR and SE images were extracted.

Tissue-type segmentation of the 3D FSPGR IR images was performed by FMRIB's Automated Segmentation Tool (FAST) (21). Lesions were masked out from the white matter partial volume images. Median intensity of the white matter, gray matter, and cerebrospinal fluid (CSF) was defined on each sequence. In order to estimate normalized brain volumes from FSPGR IR images, FSL SIENAX was used (22).

\section{Statistical Analysis}

Altogether, data from 694 lesions was used in the statistical analyses. The lesions' median intensity values were calculated on FSPGR and SE sequences and were divided by the median intensity values of the white matter. These standardized intensities of the FSPGR and SE sequences were analyzed with one-sample Wilcoxon signed rank test to test whether the lesions can be differentiated from the surrounding white matter (a ratio different from 1).

In order to separate two different lesion types, K-means clustering was carried out (IBM SPSS Statistic 23, https:// www.ibm.com/products/spss-statistics) on the lesion's median intensity values. Median lesion intensities per lesion from the FSPGR, SE, and FLAIR sequences were used, the number of clusters was set to 2 ; the iterate and classify method was used, and the number of maximum iterations was set to 10. The K-means cluster algorithm creates clusters from the dataset, placing centroids in a way that the data in a given cluster have similar attributes or closeness to the centroid, whilst the distance between clusters (centroids) is maximized (23). In order to quantify how median intensity values of the two lesion type clusters differ from the normal white matter intensity profile, we employed a bootstrap-based approach using a custom-made MATLAB script. To build up a null distribution for each subject, 5,000 random intensity values were sampled from subjectwise white matter voxels. A mean distance, defined as the average ratio of all white matter voxel intensities and the reference voxel intensity was calculated for all 5,000 selected voxels. The similar distance of lesions' median intensity was compared to this null-distribution and converted to Z-scores (which we refer to later as standardized lesion distance). This way, Z-scores take individual white matter intensity profiles into account. To assess how lesion type clusters can be separated from white matter voxels, we calculated the percentage of lesions exceeding a standardized lesion distance of $Z=2.3$ for both lesion type clusters in both sequences.

Bivariate Spearman rank correlation between clinical parameters (EDSS, disease duration, number of relapses, and number of relapses since the beginning of the treatment), normalized brain volumes, and the number and volume of lesions in the clusters was calculated. All results were corrected for multiple comparison.

\section{RESULTS}

\section{Separability of Lesions From the Surrounding White Matter on T1 Weighted Images}

Our results showed that lesions on average were separable from the surrounding white matter on both FSPGR and SE images. The intensity of the lesions was lower than white matter but higher than gray matter (and CSF) (FSPGR CSF: $Z=22.82, p \leq 0.001$; SE CSF: $Z=22.8, p \leq 0.001$; FSPGR GM: $Z=13.83, p \leq 0.001$; SE GM: $Z=21.34, p \leq 0.001$; FSPGR WM: $Z=-22.81, p \leq 0.001$; SE WM: $Z=-19.1, p \leq 0.001)$. The standardized intensity of lesions on the SE and FSPGR images were different (normalized to white matter: $U=76491.5, p \leq 0.001$ ).

Albeit the intensity distributions of white matter lesions on both the SE and FSPGR images partly overlapped with the white matter intensities, the overlapping of the lesions on SE images was more expressed (Figure 1). 


\section{Grouping the Lesions Based on Their Intensity}

Based on their intensity values on the FSPGR, SE, and FLAIR sequences, the K-means clustering algorithm grouped the 694 lesions into two clusters: 224 in Cluster 1 and 470 lesions in Cluster 2 (Figures 2, 3). The median intensity of lesions on FSPGR sequence had the maximum influence in the formation of the clusters $(F=1145.97)$, whilst median intensity values

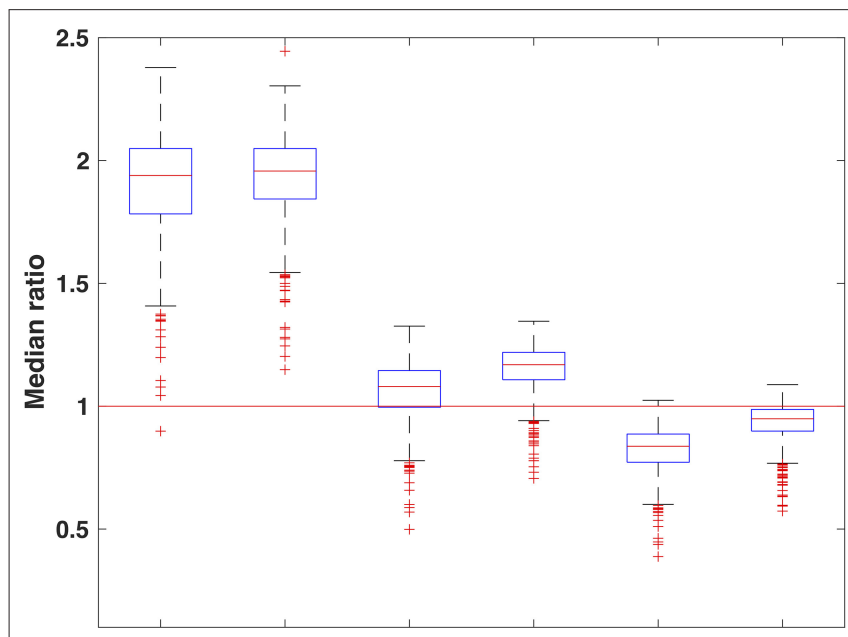

FSPGR-CSF SE-CSF FSPGR-GM SE-GM FSPGR-WM SE-WM

FIGURE 1 | Standardized intensities of lesions on FSPGR and SE images. The median intensities of lesions were divided by the median intensity of the CSF, GM, and WM, respectively. Hence, a standardized intensity value of 1 would mean lesion intensity that does not stand out from the surrounding tissue intensity.

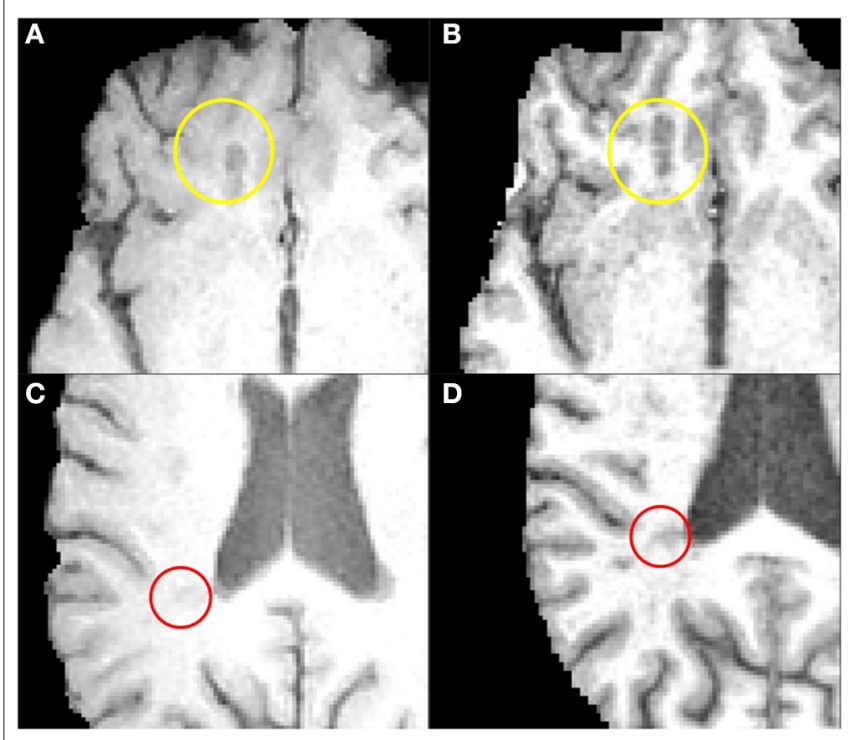

FIGURE 3 | The two clusters of lesions on FSPGR and SE images. Images on the left $(\mathbf{A}, \mathbf{C})$ are examples depict SE whilst images on the right $(\mathbf{B}, \mathbf{D})$ depict FSPGR for Cluster 1 (yellow circle) and Cluster 2 (red circle), respectively.
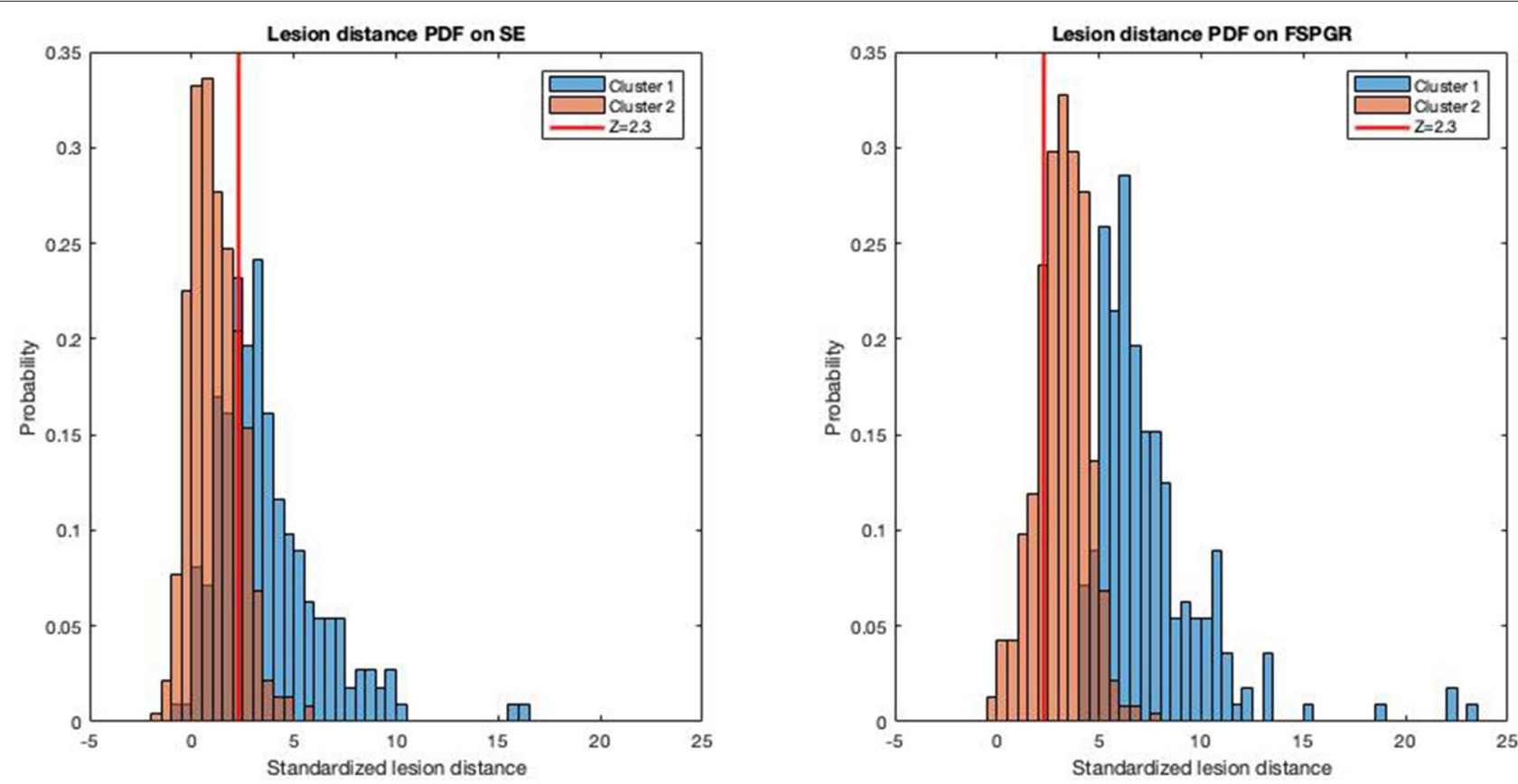

FIGURE 2 | Separability of lesion clusters from white matter intensity in FSPGR and SE sequences. Histograms depict the probability density function of standardized lesion distance (see Methods for more details) for the two lesion type clusters on FSPGR and SE sequences. The vertical red line depicts a standardized lesion distance of $Z=2$.3. PDF, probability density function. 
TABLE 2 | Summary of the results of K-means clustering.

\begin{tabular}{|c|c|c|}
\hline & 1st cluster & 2nd cluster \\
\hline Number of cases & $n=224$ & $n=470$ \\
\hline $\begin{array}{l}\text { Final cluster centers on FSPGR } \\
\text { (intensity value) }\end{array}$ & 3525.20 & 4280.42 \\
\hline $\begin{array}{l}\text { Final cluster centers on SE } \\
\text { (intensity value) }\end{array}$ & 1701.23 & 1887.77 \\
\hline $\begin{array}{l}\text { Final cluster centers on FLAIR } \\
\text { (intensity value) }\end{array}$ & 2575.23 & 2555.67 \\
\hline $\begin{array}{l}\text { Median intensity (range) on } \\
\text { FSPGR images }\end{array}$ & $3,561(2,259-3,886)$ & $4,233(3,988-4,797)$ \\
\hline $\begin{array}{l}\text { Median intensity (range) on SE } \\
\text { images }\end{array}$ & $1,732(1,142-2,022)$ & $1,902(1,717-2,090)$ \\
\hline $\begin{array}{l}\text { Median intensity (range) on } \\
\text { FLAIR images }\end{array}$ & $2,558(1,757-3,048)$ & $2,603(1,726-3,116)$ \\
\hline
\end{tabular}

The table shows the main characteristics of the two clusters of lesions derived from K-means clustering.

of lesions on FLAIR had the most negligible effect on it $(F=$ 0.74). The main characteristics of the two clusters of lesions is summarized in Table 2.

The percentage of Cluster 1 lesions exceeded a standardized lesion distance of $Z=2.3$ was $100 \%$ for the FSPGR-sequence and $69 \%$ for the SE-sequence. As for Cluster 2 lesions, $78.7 \%$ exceeded a standardized lesion distance of $Z=2.3$ on the FSPGR-sequence and $17.7 \%$ on the SE-sequence (see Figure 2 for further details). In summary, lesions in the second cluster on FSPGR images were clearly separable from the surrounding white matter; however, on SE images, the lesions were close to the mean intensity of the white matter, hence harder to visually detect.

\section{Clinical Relevance of the Lesion Types}

Spearman rank correlation values are summarized in Table 3. Our results showed that the lesion count in Cluster 2 correlated significantly with EDSS $(R=0.3, p \leq 0.042)$, with disease duration; $(R=0.33, p \leq 0.014)$, and with the normalized brain volume $(R=-0.42, p \leq 0.008)$. Also, the lesion volume of Cluster 2 showed significant correlation with EDSS $(R=0.28$, $p \leq 0.07)$ disease duration $(R=0.41, p \leq 0.007)$, and with the normalized brain volume $(R=-0.51, p \leq 0.008)$, whilst no significant correlation was found with lesion volume and lesion count of the lesions in the Cluster 1.

Total lesion volume showed significant correlation with the EDSS scores $(R=0.46, p \leq 0.007)$, and disease duration $(R=$ $0.33, p \leq 0.014)$.

Furthermore, significant correlation was found between the normalized brain volumes and EDSS scores $(R=-0.46, p \leq$ $0.008)$, disease duration $(R=-0.38, p \leq 0.008)$, number of relapses $(R=-0.36, p \leq 0.008)$, and the number of relapses since treatment onset $(R=-0.31, p \leq 0.036)$. Scatterplots depicting the significant correlations are summarized in Figure 4.

\section{DISCUSSION}

In this study, we showed that (i) T1 hypointense lesions are more easily detectable using an FSPGR sequence than an SE sequence; (ii) Almost all of the T2 hyperintense lesions were easily detectable on FSPGR, but only less than half of the lesions were easily detected using a SE sequence; (iii) Interestingly, lesions not standing out on SE sequences are the one which are clinically more important; and (iv) the very same lesions are correlated with brain atrophy.

Our results are interesting in the view of earlier investigations suggesting a stronger correlation between clinical variables and SE T1 hypointense lesions than T2 hyperintense lesions. Truyen and colleagues showed that hypointense lesion load on the SE images correlated with EDSS, but T2 hyperintense lesion load did not. Importantly, this strong correlation was only significant for secondary progressive MS (SPMS) patients (5). Enzinger presented univariate correlation between the black-hole ratio on SE (personal communication) at baseline and MS severity score 10 years later, but the association did not hold if multiple regression model was used (24). The baseline black-hole ratio was a significant predictor of conversion to secondary-progressive multiple sclerosis (SPMS), but when baseline clinical variables were inserted into the models no significant predictive value remained among the investigated MRI parameters (24). In a Multiple Sclerosis Collaborative Research Group (MSCRG) trial of interferon beta-1a, there was a significant correlation between baseline T1 lesion load (presumably SE) and EDSS, but the correlation with T2 lesion load was virtually identical (25). Similarly, in Giorgio's study, both the volume and count of baseline T1w and T2w lesions correlated with the EDSS scores, but the combination of baseline count and volume of T1w lesions predicted more precisely the 10-year EDSS worsening (8). Moreover, most of the studies describing correlation between clinical parameters and black-hole lesion load were carried out on SPMS patients $(26,27)$.

We showed that the volume and number of T1 hypointense lesions in Cluster 2 significantly correlated with the normalized brain volumes of the patients. There are only a few publications examining the relationship between $\mathrm{BH}$ and cerebral atrophy. Sailer et al. showed that an increase in T1 lesion load correlated with the increase of cerebral atrophy during an 18-month followup study (28). Paolillo et al. found significant correlation between the supratentorial brain volume and T1 total hypointense lesion load (11). Both studies suggested that the link between the volume increase of $\mathrm{T} 1$ hypointense lesions and cerebral atrophy is that local tissue damages and axonal loss might lead to increased neuronal packing density as well as contraction scarring caused by gliosis.

Our results are concordant with these former studies in that there was no correlation between Cluster 1 lesion number or volume with the clinical parameters. These are the lesions that were well differentiated on SE sequence from surrounding white matter, most comparable to traditional black-holes detected on SE images.

The importance of our findings is emphasized by Tam's studies $(16,29)$. The first study of a smaller population of mixed RR and SPMS patients showed stronger correlation between EDSS and black-hole volume when only the darkest voxels were considered (16). The consecutive study in a larger SPMS group showed the opposite, best correlation between MSFC when the brightest part of the hypointense lesions were also considered (most inclusive lesion masks) (29). These results were strengthened 
TABLE 3 | Significant correlations between clinical data and the volume and count of clustered lesions.

\begin{tabular}{|c|c|c|c|c|c|}
\hline & EDSS & Disease duration & Number of relapses & $\begin{array}{c}\text { Number of relapses since } \\
\text { treatment onset }\end{array}$ & Normalized brain volume \\
\hline CL-1 lesion count & $R=0.12, p=1.0$ & $R=0.05, p=1.0$ & $R=0.006, p=1.0$ & $R=-0.15, p=1.0$ & $R=-0.06, p=1.0$ \\
\hline CL-1 lesion volume & $R=0.14, p=1.0$ & $R=0.008, p=1.0$ & $R=0.07, p=1.0$ & $R=-0.18, p=0.77$ & $R=-0.09, p=1.0$ \\
\hline $\mathrm{Cl}-2$ lesion count & $R=0.30, p=0.042$ & $R=0.33, p=0.014$ & $R=0.02, p=1.0$ & $R=0.13, p=1.0$ & $R=-0.42, p=0.008$ \\
\hline CL-2 lesion volume & $R=0.28, p=0.007$ & $R=0.41, p=0.007$ & $R=0.18, p=0.70$ & $R=0.28, p=0.07$ & $R=-0.51, p=0.008$ \\
\hline Lesion volume (total) & $R=0.46, p=0.007$ & $R=0.33, p=0.014$ & $R=0.21, p=0.36$ & $R=0.21, p=0.38$ & - \\
\hline Lesion count (total) & $R=0.29, p=0.056$ & $R=0.28, p=0.07$ & $R=-0.002, p=1.0$ & $R=0.04, p=1.0$ & - \\
\hline Normalized brain volume & $R=-0.46, p=0.008$ & $R=-0.38, p=0.008$ & $R=-0.36, p=0.008$ & $R=-0.31, p=0.036$ & - \\
\hline
\end{tabular}

Table 2 also shows significant correlations between normalized brain volume and clinical data and the volume and count of clustered lesions (CL-1, 1st Cluster; CL-2, 2nd cluster, EDSS, Expanded Disability Status Scale). The bold values highlight the significant correlations.

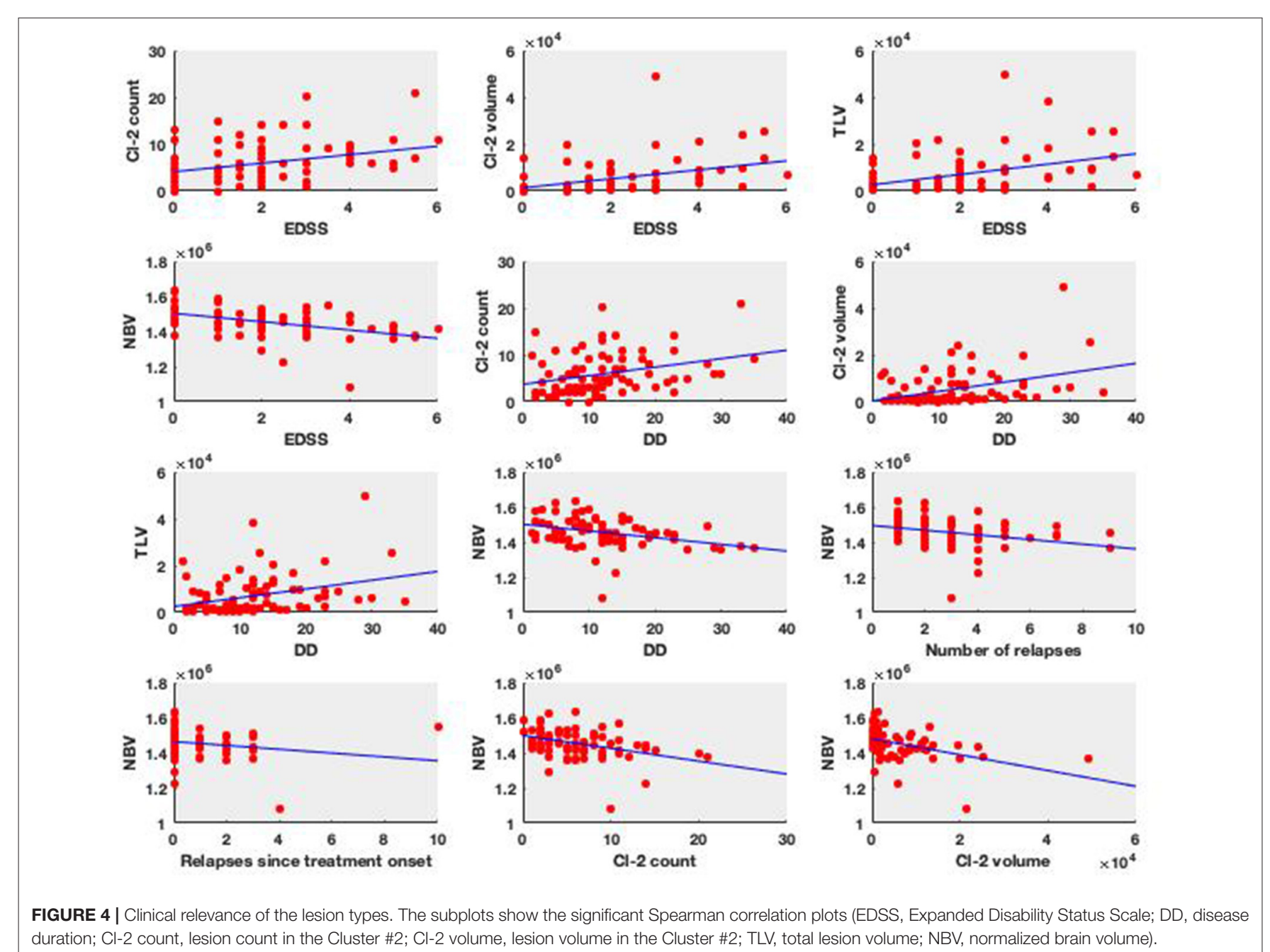

by incorporating T1 relaxation times in the analysis (3). While these studies only included SE images (personal communication), the importance of intensity variation of T1 hypointense lesions is clear. Along those lines, Adusumilli used a weighted lesion burden in which intensity values closer to CSF contribute most to the T1 hypointense lesion burden (15). Their measure shows good correlation with clinical and cognitive functions of the patients.
The importance of the intensity variation in the $\mathrm{BHs}$ is also supported by the histopathological studies. The degree of hypointensity correlated strongly with the axonal density in the lesions but not with the degree of demyelination or the number of reactive astrocytes (10). Fisher found that the contrast ratio of the T1 and MTR images (lesion intensity compared to the mean NAWM intensity) highly correlated with the axon count (9). The contrast ratios (T1, MTR, and FLAIR) differed 
significantly between myelinated and demyelinated lesions, but in the demyelinated lesions, the axon count correlated with the T1 and MTR contrast ratio, but not with the FLAIR contrast ratio. Importantly, chronic inactive lesions had the lowest contrast ratio (darkest lesions) as compared to active and chronic active lesions.

The question arises: Why did Cluster 2, with the relatively lighter lesions, show correlation with clinical measures, if the most severe tissue destruction is at the darkest part of the hypointense lesions? According to Fisher's results, the most hypointense part of the lesions are the inactive chronic lesions (9). One might speculate that these lesions are the oldest; hence, the most time was available for compensatory mechanisms and neural plasticity.

Here we used an automatic clustering approach to identify different types of lesions based on median intensity values on commonly used sequences. There were already studies to classify lesions, but some of those used images not included in the standard clinical routine (e.g., DTI, MTR, relaxometry) (30), or did not use a model-free approach (31).

Our work is not without limitations. Acquiring SE and FSPGR images with a set of different parameters would strengthen our results. The different spatial resolution of the two sequences might introduce a bias, but using these sequences we were investigating a real-life problem. Investigating various patient populations (RRMS and SPMS) and including patients with contrast-enhancing active lesion could further elaborate our findings.

\section{CONCLUSION}

Our results call attention to consider FSPGR and SE sequences together when evaluating BHs. The clinical relevance of the

\section{REFERENCES}

1. Kincses B, Hérák BJ, Szabó N, Bozsik B, Faragó P, Király A, et al. Gray matter atrophy to explain subclinical oculomotor deficit in multiple sclerosis. Front Neurol. (2019) 10:589. doi: 10.3389/fneur.2019.00589

2. Barkhof F. The clinico-radiological paradox in multiple sclerosis revisited. Curr Opin Neurol. (2002) 15:23945. doi: 10.1097/00019052-200206000-00003

3. Thaler C, Faizy T, Sedlacik J, Holst B, Stellmann JP, Young KL, et al. T1- thresholds in black holes increase clinical-radiological correlation in multiple sclerosis patients. PLoS ONE. (2015) 10:e0144693. doi: 10.1371/journal.pone.0144693

4. Bagnato F, Jeffries N, Richert ND, Stone RD, Ohayon JM, McFarland HF, et al. Evolution of T1 black holes in patients with multiple sclerosis imaged monthly for 4 years. Brain. (2003) 126(Pt 8):1782-9. doi: 10.1093/brain/awg182

5. Truyen L, van Waesberghe JH, van Walderveen MA, van Oosten BW, Polman $\mathrm{CH}$, Hommes $\mathrm{OR}$, et al. Accumulation of hypointense lesions ("black holes") on T1 spin-echo MRI correlates with disease progression in multiple sclerosis. Neurology. (1996) 47:1469-76. doi: 10.1212/WNL.47.6.1469

6. van den Elskamp IJ, Lembcke J, Dattola V, Beckmann K, Pohl C, Hong W, et al. Persistent T1 hypointensity as an MRI marker for treatment efficacy in multiple sclerosis. Mult Scler. (2008) 14:7649. doi: $10.1177 / 1352458507087842$

7. Nowaczyk N, Kalinowska-Łyszczarz A, Paprzycki W, Michalak S, Kazmierski R, Pawlak MA. Spatial distribution of white matter degenerative lesions and intensity variation of the hypointense lesion is critical. Further studies are warranted to find a standardization approach and cutoff values for the FSPGR images that could result in reducing the number of measurements (e.g., skipping the SE sequence).

\section{DATA AVAILABILITY STATEMENT}

The raw data supporting the conclusions of this article will be made available by the authors, without undue reservation.

\section{ETHICS STATEMENT}

The studies involving human participants were reviewed and approved by Regional Human Medical Ethics Committee University of Szeged, Szeged, Hungary. The patients/participants provided their written informed consent to participate in this study.

\section{AUTHOR CONTRIBUTIONS}

All authors listed have made a substantial, direct and intellectual contribution to the work, and approved it for publication.

\section{FUNDING}

This paper was supported by the National Brain Research Programs (Grant No. KTIA_13_NAP-A-II/20), by a Horizon 2020 grant (H2020-MSCA-RISE-2016 734718), an EFOP grant (EFOP-3.6.1-16-2016-00008). NS was supported by the Bolyai Scholarship Program of the Hungarian Academy of Sciences. KK was supported by the UNKP-19-3-SZTE149 New National Excellence Program of the Ministry of Human Capacities. cognitive dysfunction in relapsing-remitting multiple sclerosis patients. Neurol Neurochir Polym. (2019) 53:18-25. doi: 10.5603/PJNNS.a20 18.0001

8. Giorgio A, Stromillo ML, Bartolozzi ML, Rossi F, Battaglini M, De Leucio A, et al. Relevance of hypointense brain MRI lesions for long-term worsening of clinical disability in relapsing multiple sclerosis. Mult Scler. (2014) 20:2149. doi: 10.1177/1352458513494490

9. Fisher E, Chang A, Fox RJ, Tkach JA, Svarovsky T, Nakamura K, et al. Imaging correlates of axonal swelling in chronic multiple sclerosis brains. Ann Neurol. (2007) 62:219-28. doi: 10.1002/ana.21113

10. van Walderveen MA, Kamphorst W, Scheltens P, van Waesberghe JH, Ravid R, Valk J, et al. Histopathologic correlate of hypointense lesions on T1weighted spin-echo MRI in multiple sclerosis. Neurology. (1998) 50:12828. doi: 10.1212/WNL.50.5.1282

11. Paolillo A, Pozzilli C, Gasperini C, Giugni E, Mainero C, Giuliani $\mathrm{S}$, et al. Brain atrophy in relapsing-remitting multiple sclerosis: relationship with 'black holes', disease duration and clinical disability. J Neurol Sci. (2000) 174:85-91. doi: 10.1016/S0022-510X(00) 00259-8

12. Çinar BP, Özakbaş S. Prediction of conversion from clinically isolated syndrome to multiple sclerosis according to baseline characteristics: a prospective study. Noro Psikiyatr Ars. (2018) 55:15-21. doi: 10.29399/npa.12667

13. Kincses ZT, Ropele S, Jenkinson M, Khalil M, Petrovic K, Loitfelder $\mathrm{M}$, et al. Lesion probability mapping to explain clinical deficits and 
cognitive performance in multiple sclerosis. Mult Scler. (2011) 17:6819. doi: $10.1177 / 1352458510391342$

14. Sandi D, Biernacki T, Szekeres D, Füvesi J, Kincses ZT, Rózsa C, et al. Prevalence of cognitive impairment among Hungarian patients with relapsing-remitting multiple sclerosis and clinically isolated syndrome. Mult Scler Relat Disord. (2017) 17:57-62. doi: 10.1016/j.msard.2017.06.017

15. Adusumilli G, Trinkaus K, Sun P, Lancia S, Viox JD, Wen J, et al. Intensity ratio to improve black hole assessment in multiple sclerosis. Mult Scler Relat Disord. (2018) 19:140-7. doi: 10.1016/j.msard.2017.11.020

16. Tam RC, Traboulsee A, Riddehough A, Sheikhzadeh F, Li DK. The impact of intensity variations in T1-hypointense lesions on clinical correlations in multiple sclerosis. Mult Scler. (2011) 17:949-57. doi: 10.1177/1352458511402113

17. Kincses Z, Tóth E, Fricska-Nagy Z, Füvesi J, Rajda C, Bencsik K, et al. Az MRI helye a sclerosis multiplex kezelés hatékonyságának megítélésében II.: mérési protokollok [The role of MRI in measuring the effectivity of disease modifying treatments II]. Ideggyogy Sz. (2018) 71:81-8. doi: 10.18071/isz.71.0081

18. Nyúl LG, Udupa JK. On standardizing the MR image intensity scale. Magn Reson Med. (1999) 42:1072-81. doi: 10.1002/(SICI)15222594(199912)42:6<1072::AID-MRM11>3.0.CO;2-M

19. Smith SM. Fast robust automated brain extraction. Hum Brain Mapp. (2002) 17:143-55. doi: 10.1002/hbm.10062

20. Jenkinson M, Smith S. A global optimisation method for robust affine registration of brain images. Med Image Anal. (2001) 5:143-56. doi: 10.1016/S1361-8415(01)00036-6

21. Zhang Y, Brady M, Smith S. Segmentation of brain MR images through a hidden Markov random field model and the expectation-maximization algorithm. IEEE Trans Med Imaging. (2001) 20:45-57. doi: 10.1109/42.906424

22. Smith SM, Zhang Y, Jenkinson M, Chen J, Matthews PM, Federico A, et al. Accurate, robust, and automated longitudinal and cross-sectional brain change analysis. Neuroimage. (2002) 17:479-89. doi: 10.1006/nimg.2002.1040

23. Pérez-Ortega J, Almanza-Ortega NN, Romero D. Balancing effort and benefit of K-means clustering algorithms in Big Data realms. PLoS ONE. (2018) 13:e201874. doi: 10.1371/journal.pone.0201874

24. Enzinger C, Fuchs S, Pichler A, Wallner-Blazek M, Khalil M, Langkammer $\mathrm{C}$, et al. Predicting the severity of relapsing-remitting MS: the contribution of cross-sectional and short-term follow-up MRI data. Mult Scler. (2011) 17:695-701. doi: 10.1177/1352458510394454

25. Simon JH, Lull J, Jacobs LD, Rudick RA, Cookfair DL, Herndon RM, et al. A longitudinal study of T1 hypointense lesions in relapsing MS: MSCRG trial of interferon beta-1a. Multiple Sclerosis Collaborative Research Group. Neurology. (2000) 55:185-92. doi: 10.1212/WNL.55.2.185

26. O'Riordan JI, Gawne Cain M, Coles A, Wang L, Compston DA, Tofts P, et al. T1 hypointense lesion load in secondary progressive multiple sclerosis: a comparison of pre versus post contrast loads and of manual versus semi automated threshold techniques for lesion segmentation. Mult Scler. (1998) 4:408-12. doi: 10.1177/135245859800400502

27. Masek M, Vaneckova M, Krasensky J, Danes J, Havrdova E, Hrebikova T, et al. Secondary-progressive form of multiple sclerosis: MRI changes versus clinical status. Neuro Endocrinol Lett. (2008) 29:461-6.

28. Sailer M, Losseff NA, Wang L, Gawne-Cain ML, Thompson AJ, Miller DH. T1 lesion load and cerebral atrophy as a marker for clinical progression in patients with multiple sclerosis. A prospective 18 months followup study. Eur J Neurol. (2001) 8:37-42. doi: 10.1046/j.1468-1331.2001. 00147.x

29. Tam RC, Traboulsee A, Riddehough A, Li DK. Improving the clinical correlation of multiple sclerosis black hole volume change by pairedscan analysis. Neuroimage Clin. (2012) 1:29-36. doi: 10.1016/j.nicl.2012. 08.004

30. Thaler C, Faizy TD, Sedlacik J, Bester M, Stellmann JP, Heesen C, et al. The use of multiparametric quantitative magnetic resonance imaging for evaluating visually assigned lesion groups in patients with multiple sclerosis. J Neurol. (2018) 265:127-33. doi: 10.1007/s00415-017-8683-9

31. Bonnier G, Roche A, Romascano D, Simioni S, Meskaldji DE, Rotzinger $D$, et al. Multicontrast MRI quantification of focal inflammation and degeneration in multiple sclerosis. Biomed Res Int. (2015) 2015:569123. doi: $10.1155 / 2015 / 569123$

Conflict of Interest: The authors declare that the research was conducted in the absence of any commercial or financial relationships that could be construed as a potential conflict of interest.

Copyright @ 2021 Kocsis, Szabó, Tóth, Király, Faragó, Kincses, Veréb, Bozsik, Boross, Katona, Bodnár, László, Vécsei, Klivényi, Bencsik and Kincses. This is an open-access article distributed under the terms of the Creative Commons Attribution License (CC $B Y)$. The use, distribution or reproduction in other forums is permitted, provided the original author(s) and the copyright owner(s) are credited and that the original publication in this journal is cited, in accordance with accepted academic practice. No use, distribution or reproduction is permitted which does not comply with these terms. 